\title{
PREVALENCE AND ANATOMICAL VARIATIONS IN THE MANDIBULAR LINGUAL CANAL IN A SAMPLE OF EGYPTIAN POPULATION USING CBCT: A CROSS-SECTIONAL STUDY
}

\author{
Orchidia N. El-Hadidy*, Ahmed M. Hossam El-Din**, \\ Ahmad M. Abd El Samad ${ }^{* * *}$ and Mushira Dahaba****
}

\begin{abstract}
Aim: With the development of surgical procedures, as the mandibular anterior dental implants and chin surgeries, there has been an increase in complications associated with anatomical elements in this anterior area. One of the most dangerous complications is related to bleeding caused by injured vessels associated with the lingual foramen and canal, which can cause a compression of the airway.
\end{abstract}

Methodology: Eighty-two CBCT scans for Egyptian individuals were collected from the data base available at the Department of Oral and Maxillofacial Radiology, Faculty of Dentistry, Cairo University. Scans were selected according to specific inclusion and exclusion criteria, they were interpreted by two oral radiologists and analyzed in the three planes axial, coronal and sagittal. The midline was drawn on the axial scan by a line passing through the convexity of the genial tubercle to the mental ridge. The procedure was then standardized by adjusting the slice thickness and the spacing between all cuts at $0.4 \mathrm{~mm}$. After determining the midline on the axial plane, from the sagittal plane five consecutive cuts were taken on each side of the midline, these cuts were used for the assessment of the prevalence and the number of lingual canals present.

Results: All patients in our study had at least one median lingual foramen and canal. Regarding the number of lingual foramina; twenty subjects (24.4\%) showed single lingual foramen, thirtyseven subjects $(45.1 \%)$ showed two lingual foramina, nineteen subjects $(23.2 \%)$ showed three lingual foramina, five subjects $(6.1 \%)$ showed four lingual foramina while only a single case $(1.2 \%)$ showed six lingual foramina. In males, the most prevalent number of lingual canals was two lingual canals $(40 \%)$ followed by three lingual canals $(28.6 \%)$. On the other hand, in females, the most prevalent number of lingual canals was two lingual canals (48.9\%) followed by one canal $(27.7 \%)$.

Conclusion: These anatomical landmarks in Egyptian population vary from previous as well as other studies that were conducted on different populations. Where; all of the images had at least one lingual foramen which demonstrates high prevalence of this anatomy with different variations among Egyptian population. Therefore, it is recommended to use CBCT imaging for preoperative evaluation prior to installing dental implants.

\footnotetext{
* Master degree candidate Department of Oral and Maxillofacial Radiology, Faculty of Dentistry, Cairo University. ** Associate Professor, Department of Oral and Maxillofacial Radiology, Faculty of Dentistry, MSA University. *** Associate Professor, Department of Oral and Maxillofacial Radiology, Faculty of Dentistry, Cairo University. ****Professor of Oral and Maxillofacial Radiology, \& Head of Quality Assurance Unit, Faculty of Dentistry, Cairo University.
} 


\section{INTRODUCTION}

The lingual foramina (LF) and their bony canals are located at the internal surface of the anterior region of the mandible (Von Arx et al. 2017) ${ }^{35}$, typically described to be between the mental foramina, in the interforaminal zone. The interforaminal zone is defined by its thin alveolar process and contains the region of the mandible with the four incisors, two canines and two first premolars (Tagaya et al. 2009) $)^{34}$.

In the past century surgical procedures in the lower anterior segment of the mandible including orthognathic surgeries, dental implants (which are considered as the current preferred line of treatment for the prosthetic rehabilitation of edentulous patients), bone grafting, and lowering genial spines procedures of edentulous patients have become more frequent (Assari et al. 2017 \& Aoun et al. $2017)^{4,3}$. This area in the past has traditionally been considered as a "surgical safe zone" because of the absence of important superficial nerves or vessels. However, reports of unexplained bleeding and sublingual hematoma after genioplasties and endosseous implant placement have spurred research into the content and the vascularity of the area (Kusum et al. 2015) ${ }^{24}$.

Studies conducted on cadavers have shown that branches from the sublingual and/or submental arteries go through these anatomic structures. Therefore, despite being generally considered as a safe area during performing surgical procedures, this mandibular region may present a real lifethreatening hemorrhage from the above cited arterial branches following the lingual cortical plate trauma (Longoni et al. 2007, Marzook et. al. 2019 \& Jayabalan and Muthusekhar 2020) 26,27,20.

It was reported by many authors that during implant placement in the anterior region of the mandible a wide hematoma with a large swelling of the floor of the mouth occurs which later leads to a serious upper airway obstruction; this condition being related to the lingual cortex rupture and/or perforation (Del Castillo-Pardo de Vera et al.2008, Jaju et.al.2011, Barrientos-Lezcano et.al. 2021 \& Blanc et. al. 2021) ${ }^{11,18,6,8}$.

Cone beam CT (CBCT) offers three-dimensional radiographic imaging which has enabled the detailed study of bony structures with accuracy that was previously only possible by examination of cadavers. Comparing its dose to medical CT, it has greatly reduced doses of radiation becoming a routine diagnostic tool in implant planning and in revealing any anatomical variations.

Studies that demonstrate the variability in the mandibular lingual canal and its bony canals among the Egyptian population are in sufficient as we only found two papers El Dessouky 2016 \& Ahmed DF and Abd Alsamad 2018 ${ }^{14,2}$, and the latter was made on specific age group as it included only edentulous patients. That's why; this study was conducted to detect these variations using CBCT analysis.

\section{MATERIALS AND METHODS}

Out of a total 400 scans of the mandible which were taken from the data base available at the department of Oral and Maxillofacial Radiology, Faculty of Dentistry, Cairo University and according to our inclusion and exclusion criteria a total sample of 82 scans of the mandibular anterior region and full arch mandible belonging to Egyptian individuals were chosen and examined. The scans included $\mathbf{4 7}$ female patients and $\mathbf{3 5}$ male patients, with the age of 18 or above, both dentition and partially edentulous conditions. In addition, the scans had to show either the anterior region (from second premolar to second premolar on the other side) or the whole mandible. Included scans had to be of a good quality and their voxel resolution $0.4 \mathrm{~mm}$ to ensure the accurate detection and interpretation of the scans.

Exclusion criteria was, the absence of patients' records status and data such as name, sex, or age, presence of any deformities of the mandibles, any pathologic conditions or impacted teeth in the anterior region of the mandible, history of 
fracture and previous iatrogenic manipulation of the mandible that altered the anatomical structures. CBCT images were obtained from the available CBCT scans of Egyptian patients which were taken as a part of their dental examination, diagnosis or treatment planning during the years 2017-18-19. This was done to avoid exposing the patients to unneeded radiation dose for the research purpose, and the study was approved by the ethics committee of Cairo University in 2019.

Retrospective data analysis was performed after CBCT images were collected from the computer database. All CBCT images were acquired in a digital DICOM format. The images were then imported to Planmeca Romexis ${ }^{\circledR}$ Viewer (Romexis version 6.0.1.812; Planmeca OY, Helsinki, Finland) the slice thickness and the spacing between slices were standardized to be $0.4 \mathrm{~mm}$ in the three orthogonal planes. All measurements were done independently by two oral and maxillofacial radiologists (with different experiences). The first investigator (main researcher) performed the measurements twice on two different sessions with a time period of two weeks between the two attempts. A session including all investigators was set to agree on exact anatomic determination prior to image analysis.

\section{The presence and number of median lingual canals}

On the axial image, the midline was determined as the line passing through the convexity of genial tubercle to the mental ridge (pogonion). On the sagittal image, five consecutive cuts were taken on each side of the midline; these cuts were used for the assessment of the presence and number of median lingual canals.

\section{RESULTS}

Wide range of variations in the lingual foramen and its canals were detected while observing the scans. In males; the most prevalent number of lingual canals was two lingual canals $(\mathbf{4 0 \%})$ followed by three lingual canals $\mathbf{( 2 8 . 6 \% )}$ while the least prevalent number was six lingual canals $(\mathbf{2 . 9 \%})$.
In females; the most prevalent number of lingual canals was two lingual canals $\mathbf{( 4 8 . 9 \% )}$ ) followed by one canal $(\mathbf{2 7 . 7 \%})$ while the least prevalent number was four lingual canals $(\mathbf{4 . 3 \%})$. None of the females had six lingual canals. As regards the whole sample; the most prevalent number of lingual canals was two lingual canals $\mathbf{( 4 5 . 1 \% )}$ ) followed by one canal (24.4\%) while the least prevalent number was six lingual canals (1.2\%) (Table 1 \& Fig. 1).

TABLE (1): Descriptive statistics for number of lingual canals in the study participants

\begin{tabular}{ccccccc}
\hline \multirow{2}{*}{$\begin{array}{c}\text { Number of } \\
\text { lingual canals }\end{array}$} & $\begin{array}{c}\text { Males } \\
(\mathrm{n}=35)\end{array}$ & \multicolumn{2}{c}{$\begin{array}{c}\text { Females } \\
(\mathrm{n}=47)\end{array}$} & \multicolumn{2}{c}{$\begin{array}{c}\text { Total } \\
(\mathrm{n}=82)\end{array}$} \\
\cline { 2 - 7 } & $\mathrm{N}$ & $\%$ & $\mathrm{~N}$ & $\%$ & $\mathrm{~N}$ & $\%$ \\
\hline One & 7 & 20 & 13 & 27.7 & 20 & 24.4 \\
Two & 14 & 40 & 23 & 48.9 & 37 & 45.1 \\
Three & 10 & 28.6 & 9 & 19.1 & 19 & 23.2 \\
Four & 3 & 8.6 & 2 & 4.3 & 5 & 6.1 \\
Six & 1 & 2.9 & 0 & 0 & 1 & 1.2 \\
\hline
\end{tabular}

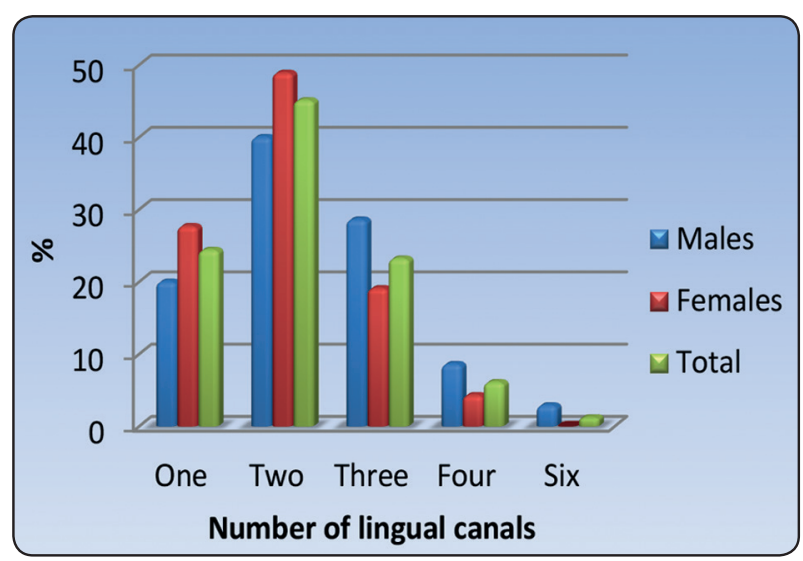

Fig. (1). Bar chart representing distribution of number of lingual canals in males, females and the whole sample 


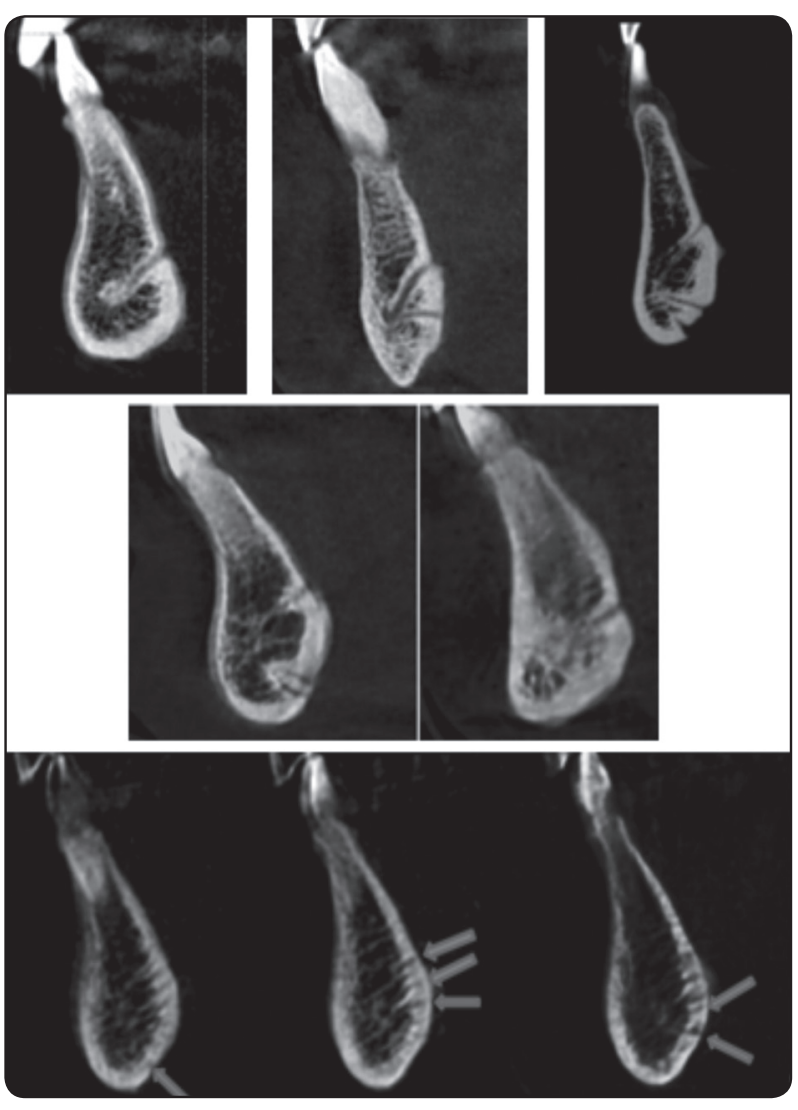

Fig. (2) CBCT images showing morphology of lingual canal

\section{DISCUSSION}

\section{Presence of lingual foramina and lingual canals}

In this study, upon examining the CBCT scans for the median mandibular region the presence of at least one lingual foramen and canal in all the patients was reported. Our study results support those of Katakami et. al.200921, Babiuc et al.2011, Sheikhi et al. 2012, Wang et al. 2015, Kumar et. al. 2017, Bhadouria et. al 2018, İşman et.al. 2021 \& Jan et al. 2021) $)^{5,34,37,23,7,16,19}$ as their studies had similarly reported at least one lingual foramen in $100 \%$ of the examined cases. Approaching almost similar results, De Andrade Santos et. al. $2021^{10}$ made a research on Brazilian subpopulation including 303 scans, two scans did not show the presence of any foramina, while 301 scans did, indicating a prevalence rate of $99.3 \%$, same results were reported by Salinas-Goodier et al. $2015^{30}$ on a Spanish subpopulation.

Some other studies have shown close results as, Kawai et. al. 2007 ${ }^{22}$ who examined 68 dry Japanese mandibles using CBCT and reported the presence of at least one foramen in $97 \%$ of the cases, similarly, Rathore et. al. $2018^{29}$ examined a sample of Indian subpopulation of 100 patients and got same results, also, Do Carmo Oliveira et. al. 2018 made a study on a Brazilian subpopulation including 202 scans and he found the lingual foramen in $97 \%$ of the cases examined.

Yet, Aoun et. al. $2017^{3}$ reported that 84 out of 90 CBCT $(93.33 \%)$ scans made on Lebanese population showed at least one lingual foramen, this difference may be due to ethnicity reason. Moreover Jacobs et al. 2002 ${ }^{17}$ also reported that the foramen was detected in only $82 \%$ of the cases that were examined by spiral CT. One possible interpretation for this disagreement is the imaging technique used by Jacobs et al.; as in our study, the thickness cut was set to $0.4 \mathrm{~mm}$ and an interval of $0.4 \mathrm{~mm}$ between the cuts unlike the $1 \mathrm{~mm}$ thickness used by Jacobs et al., which may have masked smaller diameter foramina on the mandibular midline. Another reason for this difference could be due to ethnicity reason.

\section{Number of lingual foramina}

In our present study the number of lingual canals ranged from one to six canals, with $\mathbf{2 4 . 4 \%}$ showing single canal, $\mathbf{4 5 . 1 \%}$ which was the most frequent showing two canals, $\mathbf{2 3 . 2 \%}$ showing three canals, 6.1\% showing four canals and only one male patient had six canals $\mathbf{1 . 2 \%}$.

The study results were in accordance with those of Sheikhi et al. 2012 on Isfahanian subpopulation, Choi et al. $2013^{9}$ on Korean subpopulation, Abesi 2015 et. al. ${ }^{1}$ on Iranian subpopulation, Assari et. al. $2017^{4}$ on Saudia Arabian subpopulation, Jan et. al. $2021^{19}$ on Indian subpopulation \& De Andrade Santos et. al. $2021^{10}$ on Brazilian subpopulation, 
who all noticed that double foramina are the most frequent $52.9 \%, 40 \%, 53 \%, 38.8 \%, 57.69 \%$ and $47.5 \%$, respectively. But these results are in disagreement with the findings of Kawai et. al. $2007^{22}$ on Japanese sub population, Liang et al. $2009^{25}$ on Belgian population, Babiuc et al. $2011^{5}$ on Romanian subpopulation, Sekerci et al. 2014 ${ }^{33}$ on Turkish subpopulation, Mattew et. al. $2015^{28}$ on Chinese subpopulation and Bhadouria et al. $2018^{7}$ on Indian subpopulation, who reported that single foramina is the most frequent reported finding with percentages of $47 \%, 41 \%, 71.9 \%, 48 \%, 43 \%$ and $65 \%$, respectively. This difference may be due to ethnicity reason or to the limited samples' size of some of these previously mentioned studies.

Compared to our study, some studies reported a significantly high percentage for three lingual canals, such as Wang et. al. $2015^{37}(27.7 \%)$, Zhang et. al. $2018^{39}(30.7 \%)$ and Sanhueza et. al. $2018^{32}$ (34.6\%). While Assari et. al 2017 ${ }^{4}$ detected three lingual canals in $26.9 \%$ of the participants. Among other studies less prevalence three lingual canals was reported, where, Aoun et al. 2017 $7^{3}$ reported only one case (1.1\%), Denny et al. 2016 ${ }^{13}$, two cases (1.7\%), Sanchez-Perez et al. 2018 ${ }^{31}$, four cases (4.08\%), and Xie et al. $2019^{38}, 21$ cases (2.1\%) from the total sample.

Interestingly, Babiuc et. al. $2011^{5}$, Sheikhi et al. 2012 ${ }^{34}$ Choi et al. $2013^{9}$ and El Dessouky 2016 ${ }^{14}$ detected 4 foramina, respectively, 2 out $36(3.1 \%)$, 3 out of $102(2.9 \%), 3$ out of $20(15 \%)$ and 3 out of $100(3 \%)$ mandibles assessed. Moreover, five lingual foramina were observed by Gahleitner et al. $2001^{13}$ in a single case out of 32 patients $(3 \%)$, and six foramina were found in $8.7 \%$ of the cases in a study that was conducted by Demiralp et. al. $2017^{12}$ on the ancient Anatolian population.

In our study comparing the number of canals according to the gender it was found that, in males; the most prevalent number of lingual canals was two lingual canals (40\%), which was similar to a study made on Isfahan subpopulation by Sheikhi et. al. $2012^{34}$ (47.5\%), also, Salinas Goodier et. al. $2015^{30}$ on a Spanish subpopulation reported equal prevalence for both single and double lingual canals (41.6\%) for each. On the contrary to our study which stated that the prevalence of single canal was $20 \%$, other studies stated that single lingual canal was the most prevalent in males; Denny et. al. $2016^{13}$ (70.2\%), Bhadouria et al. 2018 $(65 \%)$ \& De Andrade Santos et. al 2021 ${ }^{10}$ (52.9\%). Three lingual canals had a higher prevalence than single lingual canals in our study $\mathbf{( 2 8 . 6 \% )}$, which was a higher prevalence compared to other studies, Sekerci et. al. $2014^{33}$ ( $\left.10.6 \%\right)$, Abesi et. al. ${ }^{1} 7.5 \%$, De Andrade Santos et. al. $2021^{10}$ (4.9\%), Jan et. al. $2021^{19}(5.77 \%)$. While the least prevalent number detected in males was six lingual canals (2.9\%) similar to Wang et. al. $2015^{37}(2.0 \%)$.

On the other hand, in females; the most prevalent number of lingual canals was two lingual canals (48.9\%), similar results were approached by $\boldsymbol{D e}$ Andrade Santos et. al $2021^{10}(50.3 \%)$ \& Rathore et. al. 2021 29 (48\%). Oppositely, it was stated that single canals had higher prevalence in females by Denny et. al. 2016 ${ }^{13}$ (33\%) \& Bhadouria et al. $2018^{7}(64.7 \%)$, which doesn't support our results where the prevalence of one canal was $(27.7 \%)$. While, the least prevalent number in our study regarding females was four lingual canals (4.3\%), nearly similar result was reported by Wang et. al. $2015^{37}$ (2.0\%). Moreover, none of the females had six lingual canals, similarly to Wang et. al. $2015^{37}$, where in his study only six lingual canals were detected in males.

\section{CONCLUSION}

From the present work, the following specific conclusions could be reached:

All patients, investigated in the current study, showed at least one lingual foramen; with the most prevalent number of lingual canals being two lingual canals $(45.1 \%)$. 
The comparable gender analysis performed in the current study revealed equivocal findings between males and females regarding number.

From the high prevalence of foramina and canals in our study and being a paramount topic of discussion, the following generic conclusion could be reached:

Determination of the prevalence and the number of median lingual canals before implant surgery in mandibular midline region is crucial as these canals are constant anatomical landmarks, and with the contraption of CBCT, we can depict these canals in great details. CBCT could act as a guide to prevent damage any neurovascular bundles and should be considered before planning any surgical procedures in this area.

\section{REFRENCES}

1. Abesi, F., M., Haghanifar S and Sohanian S. (2015): Assessing the anatomical variations of lingual foramen and its bony canals with CBCT. J. of O. and Maxillofacial Radio., 20:220-227.

2. Ahmed, D.F. and Abd Alsamad, A.M. (2018): Assessment of Anatomical Variations of Median Lingual Canals: A CBCT study. Egy. Dent. J., 64: 277-285.

3. Aoun, G., Nasseh, I., Sokhn, S. and Rifai, M. (2017): Lingual foramina and canals of the mandible: anatomic variations in a lebanese population. J. of Clin. Imag. Sci., 7: 15-17.

4. Assari, A., Almashat, H., Alamry, A. and Algarni, B. (2017): Prevalence and location of the anterior lingual foramen: A cone-beam computed tomography assessment. SAU. J. of O. Sci., 4: 41-45.

5. Babiuc, I., Tar ngeanu, I. and Pauna, M. (2011): Cone beam computed tomography observations of the lingual foramina and their bony canals in the median region of the mandible. Rom. J. Morphol. Embryol., 52: 827-829.

6. Barrientos-Lezcano, F.J., Corchero-Martín, G., GonzálezNúñez, A.B. and Soler-Presas, F. (2021): Life-threatening sublingual hematoma after mandibular implant placementA case report. J. of Annalas Maxillofacial Surg.

7. Bhadouria, P., Payak, A.S., Jaju, P.P. and Shrivastava, A. (2018): Detection of lingual vascular canal by cone-beam computed tomography. Journal of Dental Implants. 1:8-20.
8. Blanc, O., Krasovsky, A., Shilo, D., Capucha, T. and Rachmiel, A. (2021): A life-threatening floor of the mouth hematoma secondary to explantation attempt in the anterior mandible. Quintessence Int., 1:66-71.

9. Choi, D.Y., Woo, Y.J., Won, S.Y., Kim, D.H., Kim, H.J and $\mathrm{Hu}, \mathrm{K} . \mathrm{S}$. (2013): Topography of the lingual foramen using micro-computed tomography for improving safety during implant placement of anterior mandibular region. J. of C.facial Surg., 24: 1403-1407.

10. De Andrade Santos, J.B., De Brito, F.C. and De Chaves, E.C. (2020): Prevalence of lingual foramina in the anterior mandible: A cone-beam computed tomography study. J. of O. \& Maxillofacial Radio., 8:10-15.

11. De Vera, J.D., Calleja, J.L.A. and Burgueño-García, M. (2008): Hematoma of the floor of the mouth and airway obstruction during mandibular dental implant placement: a case report. O. Maxillofacial Surg., 12: 223-226.

12. Demiralp, K.O., Bayrak, S., Orhan, M., Alan, A., Cakmak, E.K. and Orhan, K. (2018): Anatomical characteristics of the lingual foramen in ancient skulls: a cone beam computed tomography study in an Anatolian population. Folia Morphologica, 77: 514-520.

13. Denny, C.E., Natarajan, S., Ahmed, J., Binnal, A. and Jindal, R. (2016): Anatomic variation in lingual foramen: A cone beam computed tomography study. World J. of Dent., 7: 179-181.

14. El Dessouky Sh. Cone Beam Computed Tomographic Assessment Of The Anatomical Characteristics Of The Lingual Foramen And Its Bony Canal In The Median Region Of The Mandible In A Group Of Egyptian Patients. Egyptian Dental Journal. 2016 Jan 1;62(1-January (Part 2):363-77.

15. Gahleitner, A., Hofschneider, U., Tepper, G., Pretterklieber, M., Schick, S., Zauza, K. and Watzek, G. (2001): Lingual vascular canals of the mandible: evaluation with dental CT. J. of Radio., 220: 186-189

16. Isman, O., Kayar, S., Surmelioglu, D., Ciftci, ME. and Aktan, A.M. (2020): Evaluation of the relationship between appearances of the lingual foramen on panoramic radiography and cone-beam computed tomography. Nigeri. J. of Clini. Pract., 23:205-211.

17. Jacobs, R., Mraiwa, N., VanSteenberghe, D., Gijbels, F. and Quirynen, M. (2002): Appearance, location, course, and morphology of the mandibular incisive canal: an assessment on spiral CT scan. D.maxillofacial Radio. J., 31: 322-327 
18. Jaju, P. and Jaju, S. (2011): Lingual vascular canal assessment by dental computed tomography: a retrospective study. Ind. J. of Dent. Res., 22: 232.

19. Jan, T., Chalkoo, A.H., Begum, S. and Nazir, N. (2020): Morphometric analysis of lingual foramen on CBCT: A retrospective radiographic study. J. of O. Med., O. Surg., O. Path. and O. Radio., 6:193-8.

20. Jayabalan, J., Muthusekhar, M.R. and Muthukrishnan, A. (2020): Prevalence of Variations in the Lingual ForaminaA CBCT Study. J. of Comp. Med. Research, 11:195-202

21. Katakami K, Mishima A, Kuribayashi A, Shimoda S, Hamada Y, Kobayashi K. Anatomical characteristics of the mandibular lingual foramina observed on limited conebeam CT images. Clinical oral implants research. 2009 Apr;20(4):386-90.

22. Kawai, T., Asaumi, R., Sato, I., Yoshida, S. and Yosue, T. (2007): Classification of the lingual foramina and their bony canals in the median region of the mandible: cone beam computed tomography observations of dry Japanese mandibles. J. of O. Radio., 23: 42-48.

23. Kumar, A., Patil B. M.,Kumar E. (2017): Anatomical Variations Of Lingual Foramen And It' S Bony Canals With Cone Beam Computerised Tomography In South Indian Population - A Cross Sectional Study. Int. J. of Inno. Res. and Adv. stud., 4: 534-540.

24. Kusum, C.K., Mody, P.V., Indrajeet, D.N., Rao, S.K. and Wankhade, B.G. (2015): Interforaminal hemorrhage during anterior mandibular implant placement: An overview. Dent. Res. J., 12: 291.

25. Liang, X., Jacobs, R., Corpas, L.S., Semal, P. and Lambrichts, I. (2009): Chronologic and geographic variability of neurovascular structures in the human mandible. Foren. Sci. Int., 190: 24-32.

26. Longoni, S., Sartori, M., Braun, M., Bravetti, P., Lapi, A., Baldoni, M. and Tredici, G. (2007): Lingual vascular canals of the mandible: the risk of bleeding complications during implant procedures. J. of Impl. Dent., 16: 131-138.

27. Marzook, H.A., El-Gendy, A.A. and Darweesh, F.R., (2019): Median Perforating Canal in Human Mandible. J. of Craniofacial Surg., 30:430-432.

28. Mathew AK, Shenai P, Chatra L, Rao PK, and Prabhu RV. (2015): Computed tomographic assessment of lingual vascular channels in the mandible - An imaging study. South East Asian, J. of Clini. Res. 1:5-11.
29. Rathore, N.S., Aggarwal, A.J.N.K.A. and Tyagi, H. (2018): CBCT as a Diagnostic Tool in Visualizing Lingual Foramen: A Case Study. J. of Research and advan. in Dent., 2: 276-279.

30. Salinas-Goodier, C., Manchón, Á., Rojo, R., Coquerelle, M., Sammartino, G. and Prados-Frutos, J.C. (2016): Prevalence and location of accessory foramina in the human mandible. J. of O. Radio., 32:72-78.

31. Sanchez-Perez A, Boix-Garcia P, Lopez-Jornet P. Conebeam CT assessment of the position of the medial lingual foramen for dental implant placement in the anterior symphysis. Implant dentistry. 2018 Feb 1;27(1):43-8.

32. Sanhueza, Á., Briner, M., Calvo, M. and Cisternas, A. (2018): The presence of lingual foramina and canals on CBCT on patients over 18 years of age. Int. J. Morphol., 36:80-86

33. Sekerci, A.E., Sisman, Y. and Payveren, M.A. (2014): Evaluation of location and dimensions of mandibular lingual foramina using cone-beam computed tomography. Surg. and Radio. Anato., 36: 857-864.

34. Sheikhi, M., Mosavat, F. and Ahmadi, A. (2012): Assessing the anatomical variations of lingual foramen and its bony canals with CBCT taken from 102 patients in Isfahan. Dent. Res. J., 9: 45-51.

35. Tagaya, A., Matsuda, Y., Nakajima, K., Seki, K. and Okano, T. (2009): Assessment of the blood supply to the lingual surface of the mandible for reduction of bleeding during implant surgery. Clini. O. Impl. Res., 20: 351-355.

36. Von Arx, T., and Scott Lozanoff. (2017): Lingual foramina and canals in: Clinical Oral Anatomy, p. 463-487. Springer, Cham., Switzerland.

37. Wang, Y.M., Ju, Y.R., Pan, W.L. and Chan, C.P. (2015): Evaluation of location and dimensions of mandibular lingual canals: a cone beam computed tomography study. Int. J. of O. and Maxillofacial Surg., 44: 1197-1203.

38. Xie L, Li T, Chen J, Yin D, Wang W, Xie Z. Cone-beam CT assessment of implant-related anatomy landmarks of the anterior mandible in a Chinese population. Surgical and Radiologic Anatomy. 2019 Aug;41(8):927-34.

39. Zhang C, Zhuang L, Fan L, Mo J, Huang Z, Gu Y. Evaluation of mandibular lingual foramina with cone-beam computed tomography. Journal of Craniofacial Surgery. 2018 Jun 1;29(4):389-94. 